\title{
4-Phenylbutyric Acid Increases GLUT4 Gene Expression through Suppression of HDAC5 but not Endoplasmic Reticulum Stress
}

\author{
Hailong Hua $\mathrm{Li} \mathrm{Li}^{b}$ Changlin Wang ${ }^{\mathrm{a}}$ Hongjuan $\mathrm{He}^{\mathrm{a}}$ Ke Mao $\mathrm{KiaO} \mathrm{Ma}^{\mathrm{c}}$ \\ Ruoyu Shic Yuri Oh ${ }^{d}$ FengWei Zhang ${ }^{a}$ Ying Lu ${ }^{e}$ Qiong Wu ${ }^{a}$ Ning Gu$^{a}$ \\ aState Key Laboratory of Urban Water Resource and Environment, School of Life Science and \\ Technology, Harbin Institute of Technology, Harbin, China; ${ }^{b}$ Division of Colorectal Surgery, The \\ Third Affiliated Hospital of Harbin Medical University, Harbin, China; 'Key Laboratory of Pu-erh Tea \\ Science of Ministry of Education, Yunnan Research Center for Advanced Tea Processing, Yunnan \\ Agricultural University, Kunming, China; ${ }^{\mathrm{d}}$ Faculty of Education, Wakayama University, Wakayama, Japan; \\ eDepartment of Geriatrics, The First Affiliated Hospital of Harbin Medical University, Harbin, China
}

\section{Key Words}

4-PBA • GLUT4 • ER stress • HDAC5

\begin{abstract}
Background: The chemical chaperone 4-phenylbutyric acid (4-PBA) has been shown to relieve endoplasmic reticulum (ER) stress. Therefore, it improves insulin sensitivity and promotes glucose metabolism in skeletal muscle. Glucose transporter type 4 (GLUT4), as a major glucose transporter protein, plays a central role in glucose metabolism. Until now, it has been unclear whether 4-PBA affects GLUT4 gene expression and thus, contributes to glucose metabolism. Methods: $\mathrm{C} 2 \mathrm{C} 12$ myotubes were treated with 4-PBA, tunicamycin or butyrate and subjected to Western blot and RT-PCR. Results: 4-PBA-treated C2C12 myotubes increased GLUT4 expression and promoted glucose metabolism. Most interestingly, GLUT4 gene expression induced by 4-PBA was not associated with ER stress even in the presence of tunicamycin, an ER stress inducer. Moreover, we also found that 4-PBA inhibited histonedeacetylase 5 (HDAC5) expression in $\mathrm{C} 2 \mathrm{C} 12$ myotubes, resulting in hyperacetylation of the histone $\mathrm{H} 3$ at the myocyte enhancer factor 2 (MEF2) binding site. This increased the binding of MEF2A to the site on the GLUT4 promoter, resulting in increased GLUT4 expression. Conclusions: Our data indicate that 4-PBA increases GLUT4 expression by acetylating the MEF2 site to increase the MEF2A binding through a mechanism that involves suppression of the HDAC5 pathway, but without involving ER stress.
\end{abstract}

H. Hu and L. Li are thought to have equal contributions.

Qiong Wu and Ning Gu 


\section{Introduction}

Recent studies have indicated that insulin resistance (IR) is a key feature of diabetes and obesity [1]. Some reports have revealed that endoplasmic reticulum (ER) stress plays a pivotal role in promoting IR. Any misfolded protein in the ER may induce ER stress [2]. To deal with this stress, cells initiate the unfold protein response (UPR) [3], which is mediated by three types of ER-transmembrane protein: inositol-requiring protein 1 (IRE1), RNAdependent protein kinase-like ER eukaryotic translation initiation factor 2 alpha kinase (PERK), and activating transcription factor 6 (ATF6) [4]. When ER stress deteriorates, these three ER-transmembrane proteins could cause IR via insulin receptor substrate/ phosphatidylinositol 3-kinase (IRS/PI3K) [5]. For instance, the UPR-induced IRE1 could trigger the c-Jun $\mathrm{N}$-terminal kinase (JNK) activity and promote the serine phosphorylation of insulin receptor substrate-1 (IRS-1) and inhibit the insulin receptor signaling, resulting in IR and aggravating diabetes [6, 7].

These results demonstrate the correlation between ER stress and IR. Some recent studies have shown that attenuation of ER stress can enhance insulin sensitivity, increase glucose uptake, and reduce glucose concentration in diabetic mice [8-10]. These studies have reported that some chemical or pharmaceutical chaperones could improve ER folding capacity and facilitate the trafficking of mutant proteins [3]. Chemical or pharmaceutical chaperones are a group of low molecular weight compounds known to stabilize protein conformation. Glucose regulated protein 78 (GRP78) is a kind of chaperone which could help unfolded protein fold [11]. GRP78 can also enhance the expression of IRE1, PERK and ATF6. PERK will promote the expression of CCAAT/enhancer-binding protein homologous protein (CHOP). When the accumulation of CHOP reaches a threshold, the apoptosis pathway will open [12] and the IRE1 and ATF6 will open the ER associated degradation (ERAD) pathway [13]. With these pathways, the chaperone GPR78 relieves the UPR-induced ER stress [11].

4-phenylbutyric acid (4-PBA) is a chemical chaperone, which could relieve ER stress [14]. 4-PBA has been used in clinical trials for cystic fibrosis and neurodegenerative diseases and approved for the treatment of patients with urea-cycle enzyme deficiency [15]. In addition, numerous studies have found that 4-PBA can improve IR in type 2 diabetes. From the literature, the treatment of obese and diabetic ob/ob mice with 4-PBA can result in normalization of hyperglycemia, restoration of systemic insulin sensitivity, and increased glucose uptake compared to that in muscle and adipose tissues from vehicle-treated controls [8]. Other report showed that 4-PBA treatment can not reduce plasma glucose in hydrocortisone sodium succinate-induced diabetic mice and Goto-Kakizaki rats which ER stress dose not play a central role [10]. These reports indicate that treatment with 4-PBA enhances insulin-stimulated glucose disposal, but only in the ER stress-induced IR animal model.

Evidence from previous research indicates that 4-PBA can relieve ER stress, and thus improve IR and promote glucose metabolism. In mammalian organisms, glucose transport across the cell membrane is mediated by the glucose transporter proteins (GLUTs) [16]. In adipose tissue and skeletal muscle, glucose transporter type 4 (GLUT4) is the major glucose transporter protein [9]. From the literature, studies indicate that the regulation of GLUT4 gene expression is at the transcriptional level [17]. There is evidence that reduced GLUT4 gene expression can cause a decrease in insulin-stimulated glucose uptake, resulting in aggravation of IR in patients with type 2 diabetes [16]. Therefore, GLUT4 plays an indispensable role in the process of glucose transport. We hypothesize that GLUT4 participates in the process in which 4-PBA attenuates ER and promotes glucose uptake. As far as we are aware, at present there is no other study that has investigated the effect of 4-PBA on GLUT4 gene expression.

The purpose of this study was to clarify whether 4-PBA manipulates GLUT4 gene expression in C2C12 myotubes. We treated these myotubes with 4-PBA and then examined the GLUT4 gene expression and glucose metabolism. In addition, we investigated the possible pathways for 4-PBA to regulate GLUT4 gene expression. 


\section{Materials and Methods}

\section{Cells Culture}

Mouse C2C12 myoblasts were maintained in Dulbecco's modified Eagle's medium supplemented with $10 \%$ fetal bovine serum, penicillin (50units $/ \mathrm{mL}$ ), and streptomycin $(50 \mathrm{mg} / \mathrm{mL})$. When cells reached confluence, the medium was transferred to the differential medium containing Dulbecco's modified Eagle's medium and $2 \%$ horse serum. This day was marked the first day of differentiation. The differential medium was changed every other day. After four additional days, the differentiated C2C12 cells had fused into myotubes.

\section{Quantitative real-time polymerase chain reaction (RT-PCR)}

Total cellular RNA from C2C12 myotubes was prepared using RNAiso (Takara, Japan). First-strand cDNA was synthesized from total RNA using PrimeScript RT reagent Kit (Takara, Japan). To quantify mRNA expression, RT-PCR was performed with the ABI7000 using the SYBR Premix Ex Taq II (Takara, Japan) according to the manufacturer's protocol. Respective primer sets (forward and reverse) were as follows: GLUT4, 5-TGCTCTCCTGCAGCTGATT-3 and 5-TTCAGCTCAGCTAGTGCGTC-3; GLUT1, 5-CTTCCTGCTCATCAATCGT-3 and 5-AGCTCCAAGATGGTGACCTT-3; CHOP, 5-GTCCTGTCCTCAGATGAAATTGG-3 and 5-GCAGGGTCAAGAGTAGTGAAGGTT-3; GRP78, 5-ACCTATTCCTGCGTCGGTGT-3 and 5-GCATCGAAGACCGTGTTCTC-3; 36B4, 5-GCTCCAAGCAGATGCAGCA-3 and 5-CCGGATGTGAGGCAGCAG-3. Levels of GLUT4, GLUT1, CHOP, and GRP78 mRNA were normalized against the amount of Beta-actin mRNA.

\section{Western blot}

C2C12 myotubes were washed with PBS and resuspended in lysis buffer. Lysates were centrifuged for $15 \mathrm{~min}$ at $4^{\circ} \mathrm{C}$ and protein contents of the supernatant were determined using DC protein assay reagents package (Bio-Rad Laboratories, CA, USA). Aliquots of the proteins were separated by $10 \%$ sodium dodecyl sulfate-polyacrylamide gel electrophoresis, and transferred to PVDF membranes purchased from Bio-Rad Laboratories (CA, USA). For Western blots, membranes were incubated with antibodies. Antibodies against GLUT4, CHOP, GRP78 and histonedeacetylase 5 (HDAC5) were purchased from Cell Signaling Technology (Danvers, MA), and anti-MyoD, anti-Myogenin from Abcam (Cambridge, MA). Antibody against Acetyl-histon H3 was purchased from Millipore (Bedford, MA), and anti-MEF2A from Santa Cruz Biotechnology (Santa Cruz, CA). Bands were visualized using an ECL plus western blotting detection system (GE Healthcare, UK).

\section{Chromatin Immunoprecipitation}

Chromatin immunoprecipitation (ChIP) assays were performed as previously described using a kit from Upstate Cell Signaling Solutions [18]. Briefly, $24 \mathrm{~h}$ after 4-PBA treatment, C2C12 myotubes were cross-linked with 1\% formaldehyde and lysed on ice. Chromatin was sheared to fragments 250-1000bp by sonication and centrifuged, and the supernatant was precleared with salmon sperm DNA/protein A agarose to yield input sample. Chromatin from 500ul of the input sample was immunoprecipitated by incubation with protein agarose A and antibodies directed against MEF2A, acetylated histone H3, or with immunoglobulin G (IgG). Precipitated complexes were reverse cross-linked by adding $0.2 \mathrm{M} \mathrm{NaCl}$ and incubating at $65^{\circ} \mathrm{C}$ for $6 \mathrm{~h}$, and the coimmunoprecipitated DNA was purified by phenol-chloroform extraction and resuspended in $\mathrm{dH}_{2} \mathrm{O}$. A 270-bp fragment corresponding to nucleotides 336 to 604 of the mouse Glut4 promoter containing the MEF2 binding site was amplified by PCR using the following primers: forward 5-CAGGCATGGTCTCCACATACAC-3; reverse 5-GGTAACTCCAGCAGGATGACA-3.

\section{Glucose uptake and glycogen synthesis assay}

C2C12 myotubes were pre-incubated with 5mM 4-PBA for $12 \mathrm{~h}$ or $24 \mathrm{~h}$. Five hours before the experiment, cells were deprived of serum in DMEM containing 5mM 4-PBA. Cells were then washed with PBS and incubated with or without $100 \mathrm{nM}$ insulin for $60 \mathrm{~min}$ in KRBH buffer containing 5.6mM glucose and $\mathrm{D}-\left[\mathrm{U}^{-14 C}\right]$ glucose $\left(3.7 \times 10^{-3} \mathrm{MBq} / \mathrm{ml}\right)$. The ice-cold $0.9 \%$ (wt/vol.) $\mathrm{NaCl}$ prior to lysis in $50 \%$ (wt/vol.) $\mathrm{KOH}$ was added to terminate the incubation. Glucose uptake and cellular glycogen were precipitated from lysates as described previously [19], and associated radioactivity was determined by liquid scintillation counting. Protein was determined using the Bradford method [20]. 
Statistical analysis

Statistical differences between groups were evaluated by Student's t-test and were considered significant when $p<0.05$.

\section{Results}

4-PBA treatment increases GLUT4 mRNA and protein expressions

We treated C2C12 myotubes with $5.0 \mathrm{mM}$ 4-PBA for 12 or $24 \mathrm{~h}$ from the seventh day of differentiation, and then measured GLUT4 mRNA and protein levels and GLUT1 mRNA level. It was noted that there was an increase in expression levels of GLUT4 at both mRNA and protein (Fig. 1B, F), but the mRNA levels of GLUT1 were not affected (Fig. 1C). Moreover, we tested the mRNA and protein levels from the expression of CHOP and GRP78 under 4-PBAtreated conditions (Fig. 1D, E, F), but there were no noticeable changes between the control and the 4-PBA-treated groups.

To confirm the effects of 4-PBA on cell differentiation, we examined both the morphological changes to the $\mathrm{C} 2 \mathrm{C} 12$ myotubes after 4-PBA treatment, and also analyzed MyoD and myogenin protein levels. The results showed no obvious change in either mRNA or protein levels of the MyoD and myogenin (Fig. 1F). The cell morphology analyses of the control and 4-PBA-treated groups were also similar (Fig. 1G).

These data suggested that $5.0 \mathrm{mM}$ 4-PBA increased GLUT4 gene expression at both mRNA and protein levels without affecting cell differentiation in C2C12 myotubes after treatment for 12 or $24 \mathrm{~h}$.

Tunicamycin-induced ER stress does not change GLUT4 gene expression; 4-PBA treatment increases GLUT4 mRNA and protein expressions under tunicamycin-induced ER stress condition

Since tunicamycin treatment can aggravate ER stress, we treated C2C12 myotubes with $0.5 \mu \mathrm{g} / \mathrm{mL}$ tunicamycin for $24 \mathrm{~h}$ from the seventh day of differentiation. The results showed that the mRNA and protein levels from the expression of CHOP and GRP78 were significantly increased in the tunicamycin treated groups (Fig. 2A, B, E), indicating that the ER stress was aggravated by tunicamycin treatment. There was no discernible difference at GLUT4 mRNA and protein levels, and GLUT1 mRNA levels (Fig. 2C, D, E).

We treated C2C12 myotubes under tunicamycin-induced ER stress condition with 5.0 $\mathrm{mM} 4$-PBA for $24 \mathrm{~h}$, and found that GLUT4 mRNA and protein levels were markedly increased compared with the group treated with $0.5 \mu \mathrm{g} / \mathrm{mL}$ tunicamycin alone (Fig. 2C, E), but GLUT1 mRNA levels were not (Fig. 2D). Meanwhile, the data showed that mRNA and protein levels of CHOP and GRP78 gene expressions were reduced compared with the group treated with $0.5 \mu \mathrm{g} / \mathrm{mL}$ tunicamycin alone (Fig. 2A, B, E).

From these data, tunicamycin-induced ER stress did not affect GLUT4 gene expression, but 4-PBA increased GLUT4 mRNA and protein levels under tunicamycin-induced ER stress.

Butyrate treatment increases GLUT4 mRNA and protein expressions

To examine the effect of HDAC inhibitor on GLUT4 mRNA and protein levels and GLUT1 mRNA levels, we treated C2C12 myotubes with $5.0 \mathrm{mM}$ butyrate for 12 or $24 \mathrm{~h}$ from the seventh day of differentiation. The data showed the similar results as observed with the 4-PBA treatment. The mRNA and protein levels of GLUT4 were significantly increased after butyrate treatment (Fig. 3A, E), but GLUT1 mRNA levels were not (Fig. 3B). The CHOP and GRP78 expressions at both mRNA and protein levels were not affected (Fig. 3C, D, E).

Thus, as a HDAC inhibitor, butyrate can also increase GLUT4 gene expression at both mRNA and protein levels which were similar to that achieved with 4-PBA treatment. 


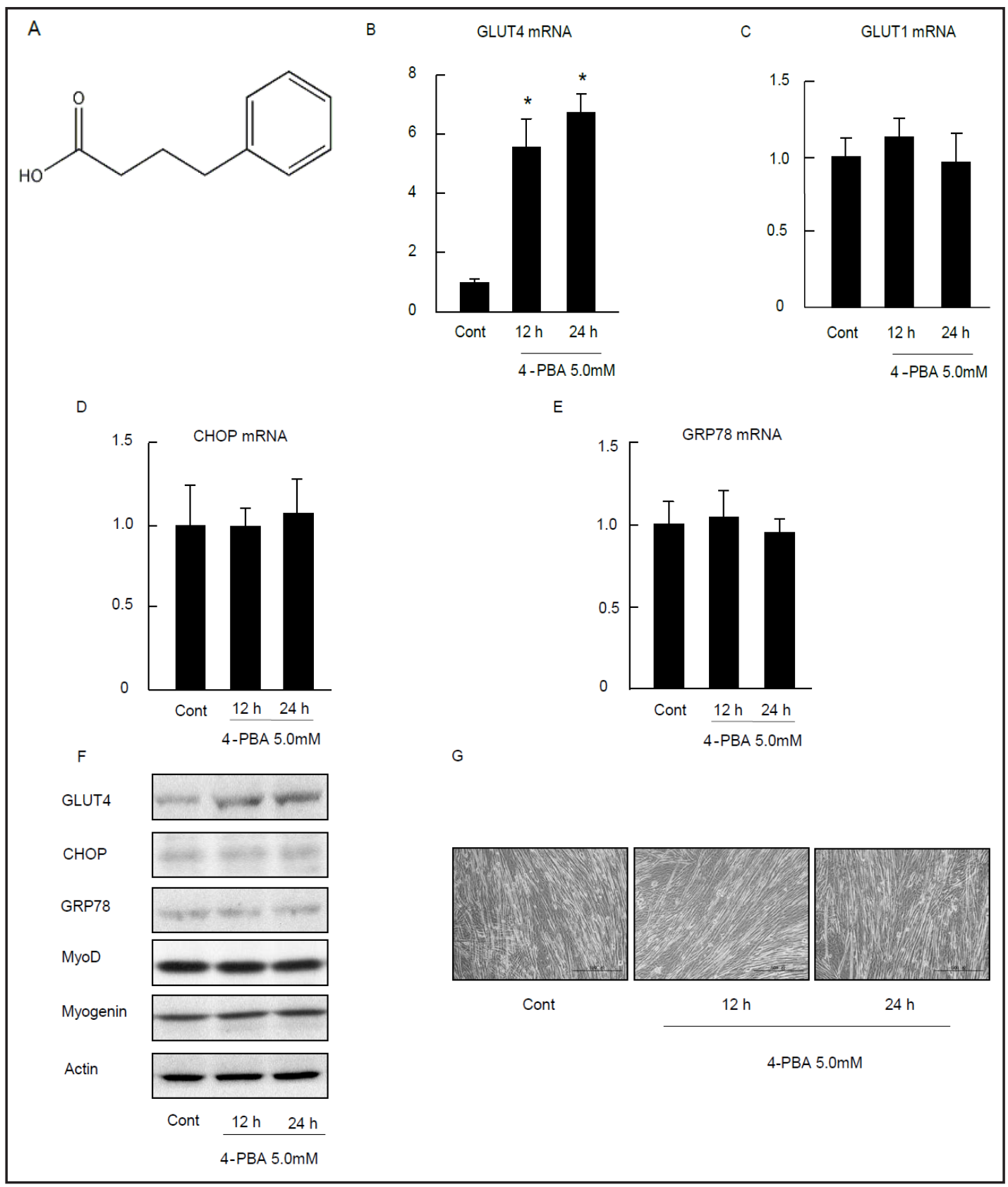

Fig. 1. Chemical structure and effects of 4-PBA on GLUT4 gene expression. Chemical structure of 4-PBA (A). C2C12 myotubes were treated with $5.0 \mathrm{mM}$ 4-PBA for 12 or $24 \mathrm{~h}$ from the seventh day of differentiation. The mRNA levels from the expression of GLUT4 were increased (B), but the mRNA levels from the expression of GLUT1(C), CHOP (D), and GRP78 (E) were not significantly altered in C2C12 myotubes after treated with 5.0 $\mathrm{mM} 4$-PBA for $12 \mathrm{~h}$ or $24 \mathrm{~h}$. The protein levels of GLUT4, CHOP, GRP78, MyoD, and myogenin were measured by Western blot (F). Cell morphology did not affect in 4-PBA-treated group (G). ${ }^{*} P<0.05$ vs. control group without any treatment. Results are mean $\pm \mathrm{SE}(\mathrm{N}=6)$.

4-PBA and butyrate treatments suppress HDAC5 protein expression and lead to induced hyperacetylation of histone $H 3$ and increased binding of MEF2A to the MEF2 site

We treated $\mathrm{C} 2 \mathrm{C} 12$ myotubes with $5.0 \mathrm{mM}$ 4-PBA or butyrate for 12 or $24 \mathrm{~h}$ from the seventh day of differentiation, and then assessed acetylation of the histone $\mathrm{H} 3$ and some 


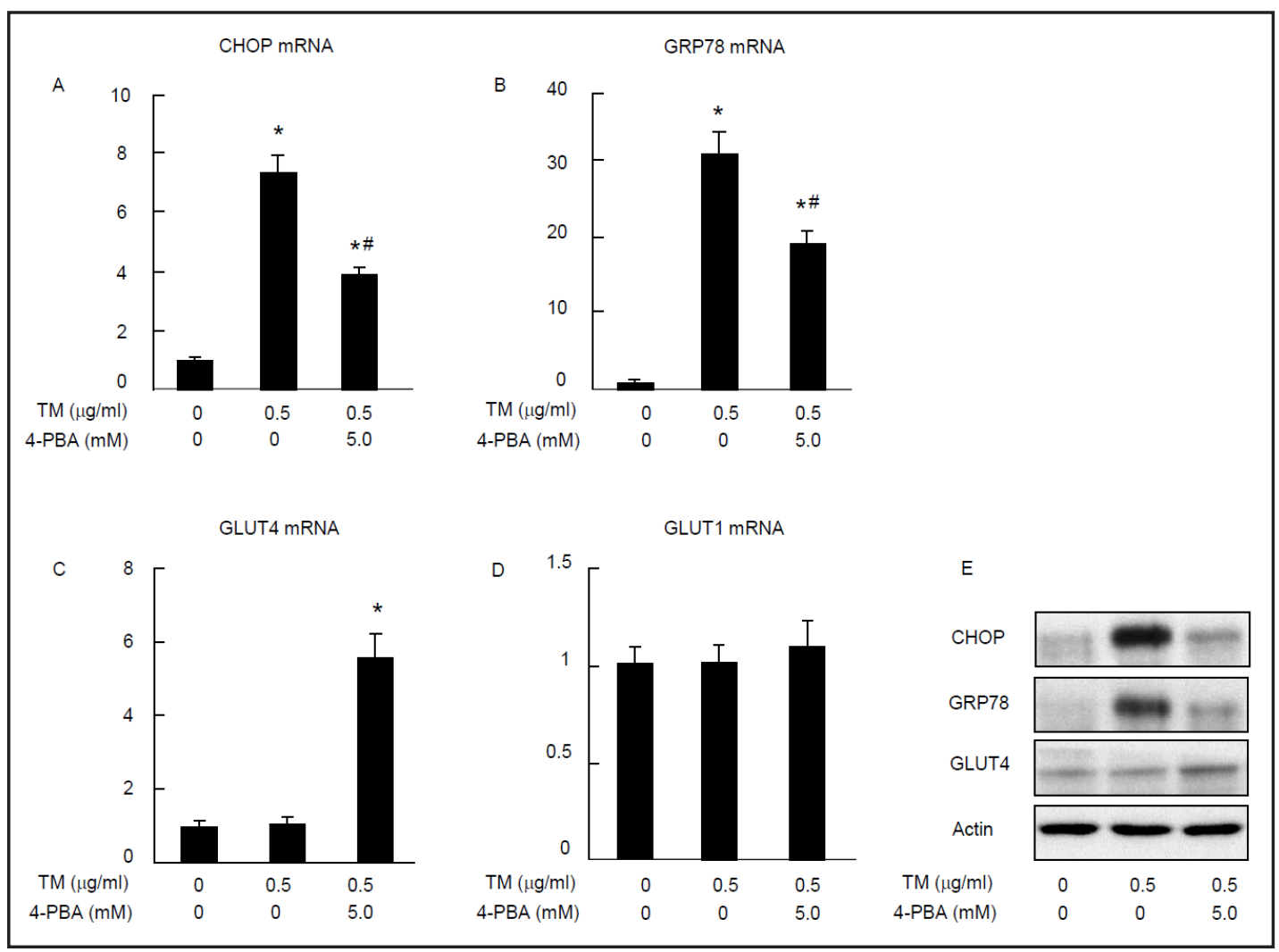

Fig. 2. 4-PBA treatment increases GLUT4 gene expression under tunicamycin-induced ER stress condition. C2C12 myotubes were treated with $0.5 \mu \mathrm{g} / \mathrm{mL}$ tunicamycin (TM) or both $0.5 \mu \mathrm{g} / \mathrm{mL}$ tunicamycin and 5.0 $\mathrm{mM}$ 4-PBA together for $24 \mathrm{~h}$ from the seventh day of differentiation. The mRNA levels from the expression of CHOP and GRP78 were increased in tunicamycin-treated group and they reduced in 4-PBA- and tunicamycin-tearted group (A and B). The mRNA levels of GLUT4 gene expression were not altered in tunicamycintreated group but increased in 4-PBA- and tunicamycin-treated group (C). The mRNA levels of GLUT1 were not altered in any group (D). The protein levels of CHOP, GRP78, and GLUT4 were measured by Western blot (E). ${ }^{*} P<0.05$ vs. control group without any treatment. \# $P<0.05$ vs. group only treated with tunicamycin. Results are mean $\pm \mathrm{SE}(\mathrm{N}=6)$.

associated protein expressions. HDAC5 protein levels were significantly reduced in the nucleus after 4-PBA and butyrate treatments, and acetylation of histone H3 increased after 4-PBA treatment or butyrate treatment (Fig. 4A). There were no differences of MEF2A protein levels between 4-PBA and butyrate treatment groups and the control group (Fig. 4A). The results of chromatin immunoprecipitation (ChIP) assay showed that 4-PBA or butyrate caused hyperacetylation of histone $\mathrm{H} 3$ in the region surrounding the MEF2 binding site on the GLUT4 gene after treated with $5.0 \mathrm{mM} 4$-PBA or butyrate for $24 \mathrm{~h}$ (Fig. 4B, D). It was also found to promote MEF2A that binds to the site in the C2C12 myotubes (Fig. 4B, C).

As a result, 4-PBA and butyrate induced hyperacetylation of histone $\mathrm{H} 3$ and increased binding of MEF2A to the MEF2 site through suppression HDAC5 protein expression.

\section{4-PBA treatment increases glucose uptake and glycogen synthesis}

We treated C2C12 myotubes with 5.0 mM 4-PBA with or without insulin for 12 or $24 \mathrm{~h}$ from the seventh day of differentiation, and then examined the glucose uptake and glycogen synthesis. In the absence of insulin, glucose uptake and glycogen synthesis did not change, but with insulin both were increased, and significantly higher in the groups treated by 4-PBA after $12 \mathrm{~h}$ and $24 \mathrm{~h}$ than in the control group (Fig. 5A, B).

These results indicated that 4-PBA treatment promotes glucose metabolism in the presence of insulin. 


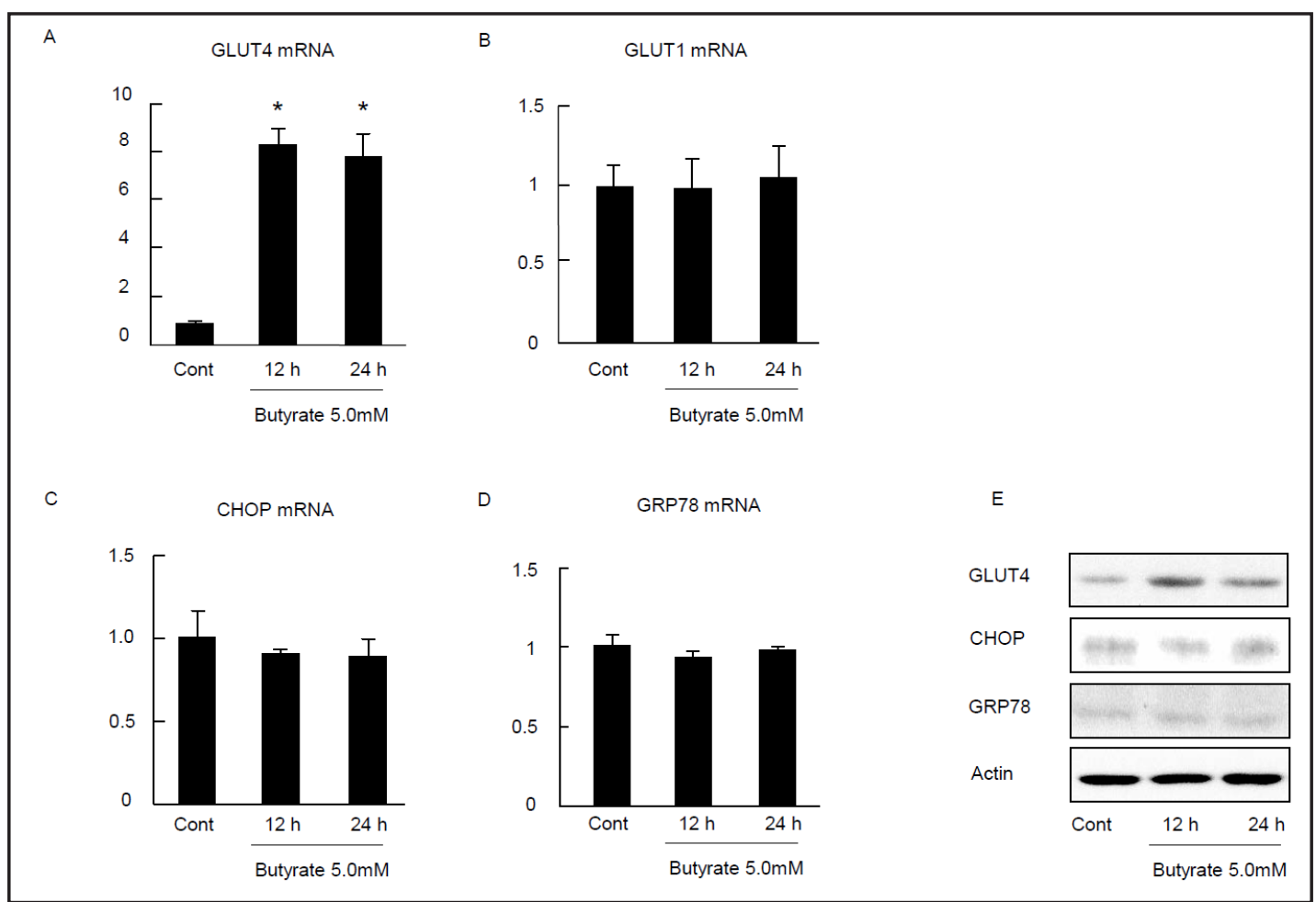

Fig. 3. Effects of butyrate on GLUT4 gene expression. C2C12 myotubes were treated with 5.0 mM butyrate for 12 or $24 \mathrm{~h}$ from the seventh day of differentiation. The mRNA levels from the expression of GLUT4 were increased (A), but the mRNA levels from the expression of GLUT1 (B) and CHOP (C), GRP78 (D) gene expressions were not significantly altered in C2C12 myotubes after treated with $5.0 \mathrm{mM}$ butyrate for $12 \mathrm{~h}$ or 24 h. The protein of GLUT4, CHOP, and GRP78 were measured by Western blot (E). ${ }^{*} P<0.05$ vs. control group without any treatment. Results are mean $\pm \mathrm{SE}(\mathrm{N}=6)$.

\section{Discussion}

In skeletal muscle cells, glucose uptake is critically dependent on GLUT4 [9]. GLUT4 gene expression is generally decreased in the skeletal muscle tissues of type 2 diabetic patients; thus, increased GLUT4 gene expression can effectively improve insulin-stimulated glucose uptake, which decreases plasma glucose levels [16]. In the present study, we treated C2C12 myotubes with $5.0 \mathrm{mM} 4$-PBA for 12 or $24 \mathrm{~h}$ from the seventh day of differentiation and then assessed GLUT4 mRNA and protein levels. The results showed that GLUT4 mRNA and protein levels significantly increased after treatment, but the mRNA levels of GLUT1 were not affected (Fig. 1B, C, F). 4-PBA treated undifferentiated myocyte and adipocyte can inhibit these cells differentiation [21, 22]. In our study, cell morphology data, MyoD and myogenin protein levels showed that 4-PBA did not affect C2C12 myotubes differentiation under the above experimental conditions (Fig. 1F, G). These findings suggest that 4-PBA might affect differentiated $\mathrm{C} 2 \mathrm{C} 12$ myotubes less than undifferentiated $\mathrm{C} 2 \mathrm{C} 12$ myocytes.

4-PBA attenuates ER stress and thus improves IR, which attenuates high plasma glucose levels in $o b / o b$ mice [8]. Previous studies have demonstrated that 4-PBA can effectively alleviate high levels of ER stress induced by tunicamycin and thapsigargin [14, 15]. We also found that $5.0 \mathrm{mM}$ 4-PBA treatment of a tunicamycin-induced ER stress group reduced mRNA and protein levels from the expression of CHOP and GRP78, which are two marker genes of ER stress (Fig. 2A, B, E). However, 4-PBA did not influence the basal mRNA and protein levels from the expression of CHOP and GRP78 in C2C12 myotubes under normal culture conditions (Fig. 1D, E, F), indicating that 4-PBA treatment did not affect basal ER stress. Thus, 4-PBA might not be able to ameliorate low levels of ER stress. 


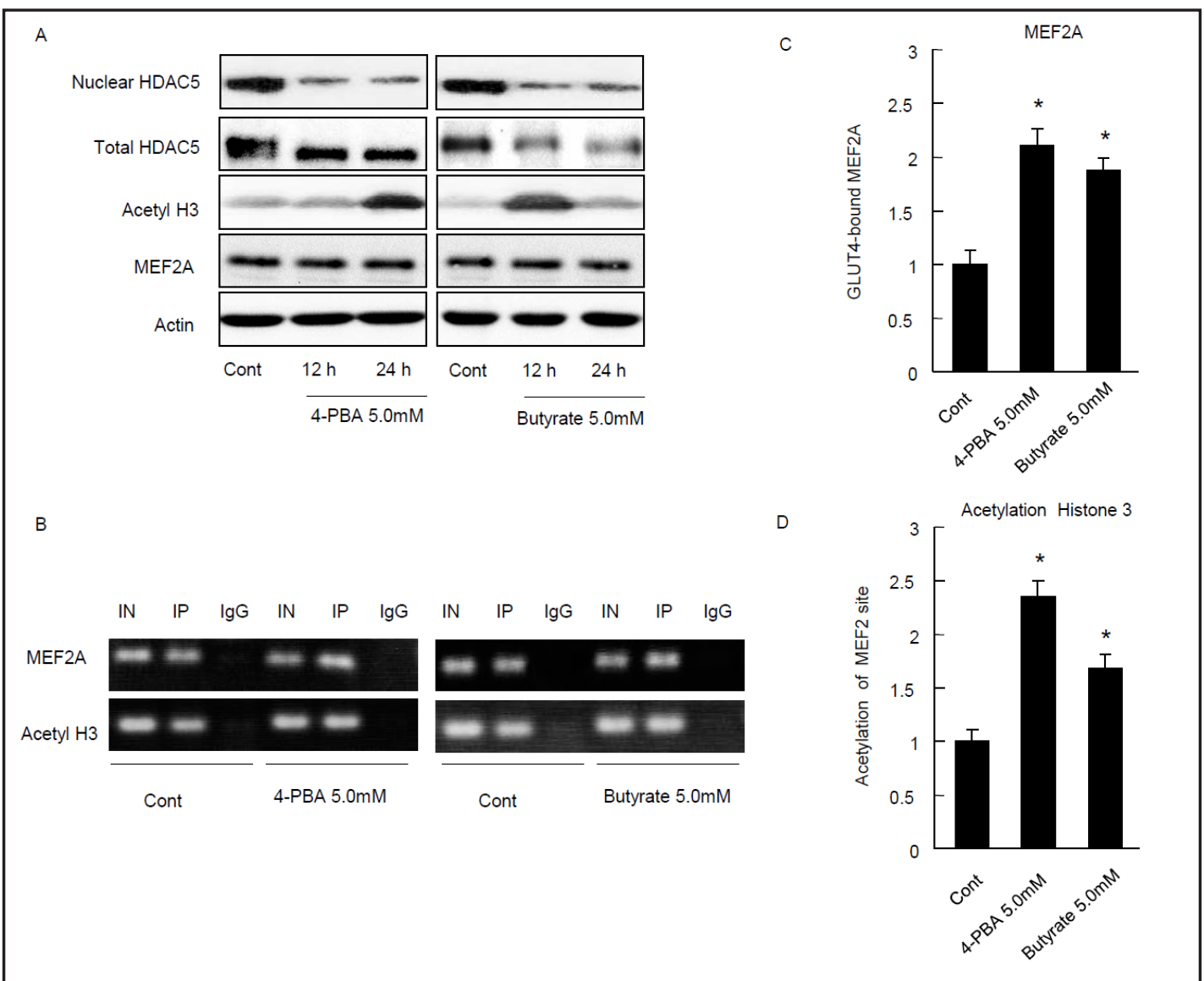

Fig. 4. 4-PBA treatment reduces HDAC5 protein expression, and induces hyperacetylation of histone H3 at the MEF2 site on the Glut4 promoter and increases the binding of MEF2A to the site. C2C12 myotubes were treated with $5.0 \mathrm{mM}$ 4-PBA or $5.0 \mathrm{mM}$ butyrate from the seventh day of differentiation for $12 \mathrm{~h}$ and $24 \mathrm{~h}$, and then the protein levels of Acetyl H3, MEF2A and HDAC5 were measured by Western blot (A). A section of the GLUT4 promoter spanning the MEF2 binding site was PCR amplified from DNA that was coimmunoprecipitated (IP) with acetylated histone H3 (Acethl H3), MEF2A antibody, or normal IgG and from corresponding input (IN) samples after C2C12 myotubes were treated with 5.0 mM 4-PBA or 5.0 mM butyrate from the seventh day of differentiation for $24 \mathrm{~h}$ (B). Relative amounts of GLTU4 promoter-bound MEF2A calculated from signal densities from PCR reactions. The bars represent the IP/IN ratio normalized to control as defined one unit (C). Relative amounts of acetylated histone H3 at the MEF2 site on the GLUT4 gene. The bars represent the IP/IN ratio normalized to control (D). ${ }^{*} P<0.05$ vs. control group without any treatment. Results are mean $\pm \mathrm{SE}(\mathrm{N}=6)$.

To determine whether 4-PBA-induced GLUT4 gene expression was associated with ER stress, we measured GLUT4 gene expression in C2C12 myotubes under low-level ER stress and tunicamycin-induced high-level ER stress. We treated C2C12 myotubes with $0.5 \mu \mathrm{g} / \mathrm{mL}$ tunicamycin for $24 \mathrm{~h}$ from the seventh day of differentiation to induce ER stress. We found that mRNA and protein expression for both CHOP and GRP78 were increased, suggesting that tunicamycin has effectively induced a high level of ER (Fig. 2A, B, E). Under this condition, the mRNA and protein levels from the expression of GLUT4 and GLUT1 were not significantly altered, indicating that tunicamycin-induced ER stress does not affect GLUT4 or GLUT1 gene expression (Fig. 2C, D, E). We then treated C2C12 myotubes with $5.0 \mathrm{mM}$ 4-PBA under tunicamycin-induced high level ER stress. The mRNA and protein levels from the expression of CHOP and GRP78 were reduced compared with the group treated with tunicamycin alone, indicating that 4-PBA relieved the high level of stress induced by tunicamycin (Fig. 2A, B). 


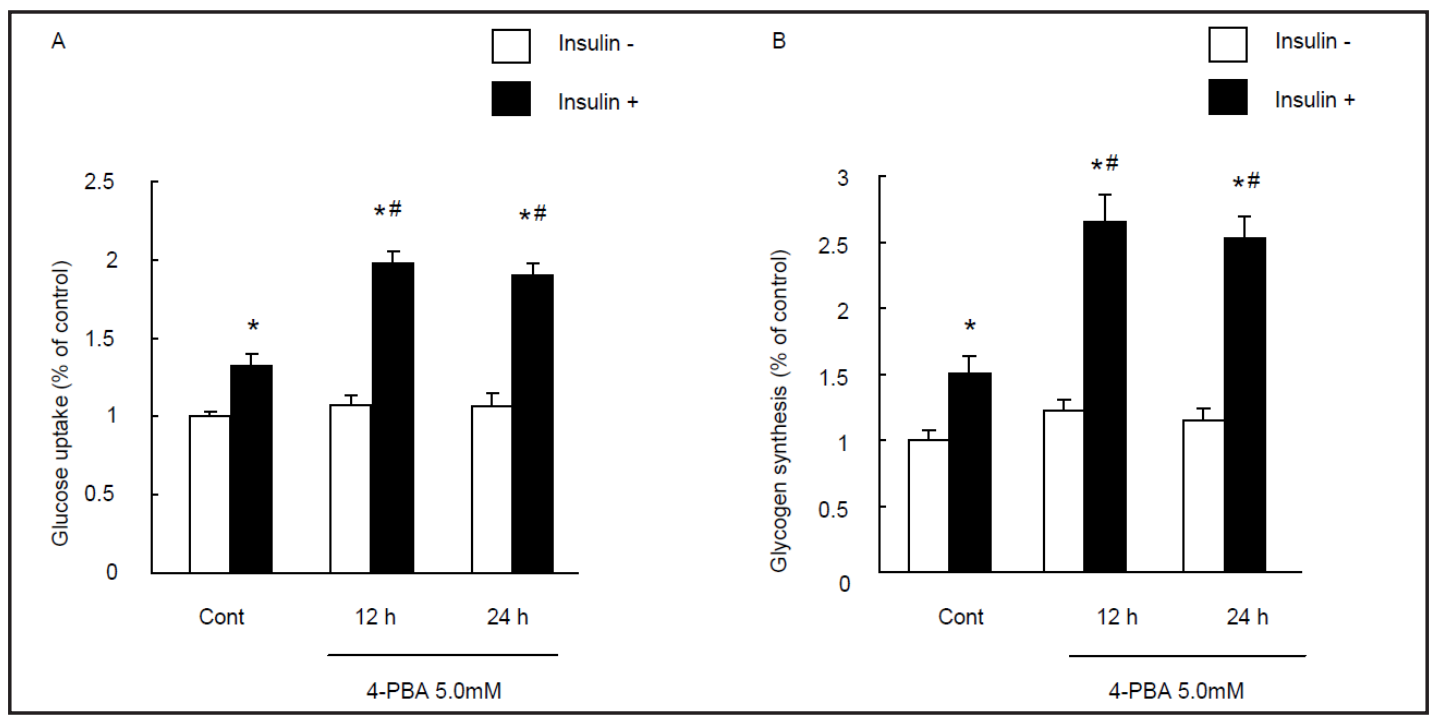

Fig. 5. 4-PBA treatment increases glucose uptake and glycogen synthesis. C2C12 myotubes were treated with 5.0 mM 4-PBA or both 4-PBA and insulin together from the seventh day of differentiation for $12 \mathrm{~h}$ and 24 h. Glucose uptake and glycogen synthesis increased in the presence of insulin in C2C12 myotubes, but glucose uptake and glycogen synthesis increased significantly in the $5.0 \mathrm{mM}$ 4-PBA-treated groups compared with the insulin-treated control groups (A and B). ${ }^{*} P<0.05$ vs. each group without insulin-treatment. \# $P<0.05$ vs. control group only treated with insulin. Results are mean $\pm \mathrm{SE}(\mathrm{N}=6)$.

Fig. 6. Schematic illustration depicting pathway that 4-PBA increases GLUT4 gene expression. 4-PBA inhibits HDAC5 gene expression, and thus induce histone $\mathrm{H} 3$ acetylation in GLUT4 promoter. The decrease of HDAC5 also induces MEF2A binding to the GLUT4 promoter. Therefore, 4-PBA increased GLUT4 gene expression and then increased glucose metabolism in the presence of insulin in C2C12 myotubes via above pathways. (up arrow means increased; down arrow means reduced).

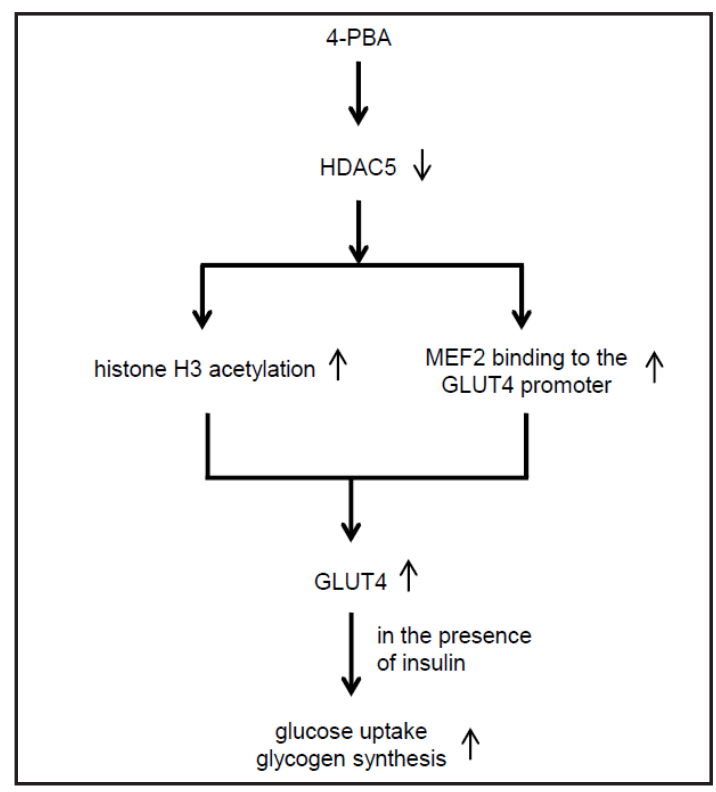

Notably, the mRNA and protein levels of GLUT4 were markedly increased compared with the group treated with tunicamycin alone (Fig. 2C, E). These results suggest that ER stress, whether aggravated or not, does not affect GLUT4 mRNA and protein levels. In addition, the data showed that 4-PBA also increased GLUT4 gene expression under tunicamycin-induced ER stress. Therefore, our findings indicate that there is no correlation between ER stress and GLUT4 gene expression and that 4-PBA increases GLUT4 gene expression via an unknown pathway that does not involve ER stress.

The previous study demonstrated that 4-PBA is a chemical chaperones that can attenuate ER stress [14]. 4-PBA also promotes chromosomal histone acetylation by reducing histone deacetylase (HDAC) activity [23]. HDACs regulate acetylation homeostasis, which is one of 
the epigenetic molecular mechanisms of cancer and cardiac dysfunction [24, 25]. Class II HDAC isoforms include HDAC4, HDAC5, HDAC7, and HDAC9. Among these, HDAC5 has been implicated as an important factor in regulating GLUT4 gene expression [26]. In fact, maximal transcriptional activation of GLUT4 is achieved when MEF2 proteins are bound to the MEF2 site, but class II HDACs, especially HDAC5, inhibit the ability of MEF2 to bind to the site [27]. There is some credible evidence that decreased HDAC5 activity increases the accessibility of MEF2 to MEF2 binding sites [17, 18]. In addition, HDAC5 induces MEF2 deacetylation at multiple lysine residues, and hypoacetylation reduces MEF2 binding activity [28]. Another study reported that with the inhibitory action of monophosphate-dependent protein kinase (AMPK) and calmodulin-dependent protein kinase (CaMK), HDAC5 separates from MEF2 and induces MEF2 binding to the promoter of the GLUT4 gene, so the suppression of HDAC5 promotes GLUT4 gene expression [17]. 4-PBA has been found to inhibit the activity of class I and II HDACs $[23,29]$. Based on this, we infer that 4-PBA increases GLUT4 gene expression through suppression of class II HDACs, especially HDAC5.

Our hypothesis was confirmed with the use of butyrate, which is a HDAC inhibitor and has been used to inhibit deacetylation in many experiments [30]. In our study, the mRNA and protein levels of GLUT4 were significantly increased in C2C12 myotubes treated with butyrate (Fig. 3A, E), but GLUT1 mRNA levels were not affected (Fig. 3B). The result was similar to that achieved with 4-PBA treatment; butyrate did not influence normal ER stress in C2C12 myotubes (Fig. 3C, D, E). These results reveal that 4-PBA-induced GLUT4 gene expression is not mediated by the ER stress pathway. In addition, we provide evidence that 4-PBA and butyrate reduced HDAC5 expression levels and that histone $\mathrm{H} 3$ remained hyperacetylated (Fig. 4A). In support of the notion that maximal GLUT4 transcriptional activation is achieved when MEF2 proteins are bound to the MEF2 site, we found increased histone H3 acetylation at the MEF2 binding site on the GLUT4 promoter after C2C12 myotubes were treated with 4-PBA or butyrate for $24 \mathrm{~h}$ (Fig. 4B, D). These findings confirm that H3 acetylation status at the MEF2 binding site on the GLUT4 promoter can be regulated by 4-PBA or butyrate. Furthermore, recent studies have demonstrated that MEF2A was acetylated at multiple lysine residues and that this acetylation increases MEF2A binding activity [28]. Indeed, we observed increased binding activity of MEF2A to the MEF2 binding site (Fig. 4B, C). These findings suggest that the increase in MEF2A binding activity may be due to 4-PBA and butyrate inhibition of HDACs, including HDAC5. The above data show that 4-PBA inhibits HDAC5 gene expression, resulting in hyperacetylation of histone $\mathrm{H} 3$ at the MEF2 binding site, which increases the binding of MEF2A to the GLUT4 promoter, leading to increased GLUT4 gene expression in C2C12 myotubes.

GLUT4 mediates glucose transport in response to insulin levels. Insulin induces the translocation of GLUT4 from intracellular storage vesicles to the plasma membrane, thus increasing glucose transport [16]. Therefore, the increased GLUT4 gene expression influences glucose metabolism [31]. After treatment with $5.0 \mathrm{mM} \mathrm{4-PBA}$ for $24 \mathrm{~h}$, GLUT4 mRNA and protein levels were increased in $\mathrm{C} 2 \mathrm{C} 12$ myotubes (Fig. 1B, F). To determine whether 4-PBAupregulated GLUT4 gene expression contributes to the glucose transport response in the presence of insulin, we compared glucose metabolism levels in 4-PBA-treated groups with or without insulin and with the control group (no treatment). We found that 4-PBA significantly increased glucose uptake and glycogen synthesis under insulin treatment (Fig. 5A, B). These results indicate that 4-PBA promotes GLUT4 gene expression and leads to increased glucose uptake and glycogen synthesis in C2C12 myotubes in the presence of insulin.

Recent studies have mainly focused on the mechanism of 4-PBA-mediated attenuation of ER stress in models of diabetes and obesity. There is no report about whether 4-PBAinduced GLUT4 gene expression can reduce plasma glucose in a diabetic model. 4-PBA can only relieve ER stress-induced IR and thus reduce plasma glucose in $o b / o b$ mice, in which ER stress plays a central role. It does not reduce plasma glucose in other diabetic models, such as hydrocortisone sodium succinate-induced diabetic mice and Goto-Kakizaki rats [10]. These results suggest that 4-PBA-mediated attenuation of ER stress-induced IR, but not 4-PBA-mediated increase of GLUT4 gene expression, plays a decisive role in 4-PBA's ability 
to decreased plasma glucose in diabetic mice.

Our results demonstrate that 4-PBA suppresses the activity of HDAC5, increases histone $\mathrm{H} 3$ acetylation at the MEF2 binding site on the GLUT4 gene, and increases MEF2A binding to the GLUT4 promoter, resulting in increased GLUT4 gene expression and glucose metabolism in C2C12 myotubes (Fig. 6). Therefore, 4-PBA promotes GLUT4 gene expression through a mechanism that involves suppression of the HDAC5 pathway but without involving ER stress.

\section{Acknowledgements}

This work was supported by the National Natural Science Foundation of China (No. 31271593; 31040054); The Natural Science Foundation of Heilongjiang Province (No. LC2012C07); Open Project of State Key Laboratory of Urban Water Resource and Environment of Harbin Institute of Technology (No. ES201115).

\section{Reference}

1 Soumaya K: Molecular mechanisms of insulin resistance in diabetes. Adv Exp Med Biol 2012;771:240-251.

2 Schröder M, Kaufman RJ: ER stress and the unfolded protein response. Mutat Res 2005;569:29-63.

3 Hoozemans JJ, Scheper W: Endoplasmic reticulum: The unfolded protein response is tangled in neurodegeneration. Int J Biochem Cell Biol 2012;44:1295-1298.

4 Gibbs EM, Stock JL, McCoid SC, Stukenbrok HA, Pessin JE, Stevenson RW, Milici AJ, McNeish JD: Glycemic improvement in diabetic $\mathrm{db} / \mathrm{db}$ mice by overexpression of the human insulin-regulatable glucose transporter (Glut4). J Clin Invest 1995;95:1512-1518.

-5 Ozawa K, Miyazaki M, Matsuhisa M, Talano K, Nakatani Y, Hatazaki M, Tamatani T, Yamagata K, Miyagawa J, Kitao Y, Hori O, Yamasaki Y, Ogawa S: The endoplasmic reticulum chaperone improves insulin resistance in type 2 diabetes. Diabetes 2005;54:657-663.

-6 Zhao L, Ackerman SL: Endoplasmic reticulum stress in health and disease. Curr Opin Cell Biol 2006;18:444-452.

7 Cnop M, Foufelle F, Velloso LA: Endoplasmic reticulum stress, obesity and diabetes. Trends Mol Med 2012;18:59-68.

8 Özcan U, Yilmaz E, Özcan L, Furuhashi M, Vaillancourt E, Smith RO, Görgün CZ, Hotamisligil GS: Chemical chaperones reduce ER stress and restore glucose homeostasis in a mouse model of type 2 diabetes. Science 2006;313:1137-1140.

-9 Christensen DP, Dahllöf M, Lundh M, Rasmussen DN, Nielsen MD, Billestrup N, Billestrup N, Grunnet LG, Mandrup-Poulsen T: Histone Deacetylase (HDAC) Inhibition as a Novel Treatment for Diabetes Mellitus. The Feinstein Institute for Medical 2011;17:378-390.

10 Xu TY, Chen RH, Wang P, Zhang RY, Ke SF, Miao CY: 4-Phenyl butyric acid does not generally reduce glucose levels in rodent models of diabetes. Clin Exp Pharmacol Physiol. 2010;37:441-446.

11 Li Z, Zhang T, Dai H, Liu G, Whang H, Sun Y, Zhang Y, Ge Z: Endoplasmic reticulum stress is involved in myocardial apoptosis of streptozocin-induced diabetic rats. J Endocrinol 2008;196:565-572.

-12 Wang XZ, Lawson B, Brewer JW, Zinszner H, Sanjay A, Mi LJ, Boorstein R, Kreibich G, Hendershot LM, Ron D: Signals from the stressed endoplasmic reticulum induce C/EBP-homologous protein (CHOP/GADD153). Mol Cell Biol 1996;16:164273-264280.

13 Casagrande R, Stern P, Diehn M, Shamu C, Osario M, Zúñiga M, Brown PO, Ploegh H: Degradation of proteins from the ER of S. cerevisiae requires an intact unfolded protein response pathway. Mol Cell 2000;5:729735.

14 Kim DS, Li B, Rhew KY, Oh HW, Lim HD, Lee W, Chae HJ, Kim HR: The regulatory mechanism of 4-phenylbutyric acid against ER stress-induced autophagy in human gingival fibroblasts. Arch Pharm Res 2012;35:269-1278. 
Hu et al.: 4-PBA Increases GLUT4 Gene Expression through Suppression of HDAC5

15 Kubota K, Niinuma Y, Kaneko M, Okuma Y, Sugai M, Omura T, Uesugi M, Uehara T, Hosoi T, Nomura Y: Suppressive effects of 4-phenyl butyrate on the aggregation of Pael receptors and endoplasmic reticulum stress. J Neurochem 2006;97:1259-1268.

16 Gaster M, Staehr P, Beck-Nielsen H, Schrøder HD, Handberg A: GLUT4 is reduced in slow muscle fibers of type 2 diabetic patients: is insulin resistance in type 2 diabetes a slow, type 1 fiber disease? Diabetes 2001;50:1324-1329.

17 Smith JA, Kohn TA, Chetty AK, Ojuka EO: CaMK activation during exercise is required for histone hyperacetylation and MEF2A binding at the MEF2 site on the Glut4 gene. Am J Physiol Endocrinol Metab 2008;295:E698-E704.

18 Smith JA, Collins M, Grobler LA, Magee CJ, Ojuka EO: Exercise and CaMK activation both increase the binding of MEF2A to the Glut4 promoter in skeletal muscle in vivo. Am J Physiol Endocrinol Metab 2007;292:E413-E420.

19 Guo H, Ling W, Wang Q Liu C, Hu Y, Xia M: Cyanidin 3-glucoside protects 3T3-L1 adipocytes against H2O2or TNFa-induced insulin resistance by inhibiting c-Jun NH2-terminal kinase activation. Biochem Pharmacol 2008;75:1393-1401.

20 Blair AS, Hajduch E, Litherland GJ, Hundal HS: Regulation of glucose transport and glycogen synthesis in L6 muscle cells during oxidative stress. Evidence for cross-talkbetween the insulin and SAPK2/p38 mitogenactivated proteinkinase signaling pathways. J Biol Chem 1999;274:36293-36299.

-21 Nakanishi K, Dohmae N, Morishima N: Endoplasmic reticulum stress increases myofiber formation in vitro. FASEB J 2007;21:2994-3003.

-22 Basseri S, Lhoták S, Sharma AM, Austin RC: The chemical chaperone 4-phenylbutyrate inhibits adipogenesis by modulating the unfolded protein response. J Lipid Res 2009;50:2486-2501.

23 Wright JM, Zeitlin PL, Cebotaru L, Guggino SE, Guggino WB: Gene expression profile analysis of 4-phenylbutyrate treatment of IB3-1 bronchial epithelial cell line demonstrates a major influence on heatshock proteins. Physiol Genomics 2004;16:204-211.

24 Baylin SB, Ohm JE: Epigenetic gene silencing in cancer - a mechanism for early oncogenic pathway addiction? Nat Rev Cancer 2006;6:107-116.

25 McKinsey TA, Olson EN: Toward transcriptional therapies for the failing heart: Chemical screens to modulate genes. J Clin Invest 2005;115:538-546.

26 Sparling DP, Griesel BA, Weems J, Olson AL: GLUT4 enhancer factor (GEF) interacts with MEF2A and HDAC5 to regulate the GLUT4 promoter in adipocytes. J Biol Chem 2008;283:7429-7437.

27 Weems JC, Griesel BA, Olson AL: Class II histone deacetylases downregulate GLUT4 transcription in response to increased cAMP signaling in cultured adipocytes and fasting mice. Diabetes 2012;61:14041414.

28 Mukwevho E, Kohn TA, Lang D, Nyatia E, Smith J, Ojuka EO: Caffeine induces hyperacetylation of histones at the MEF2 site on the Glut4 promoter and increases MEF2A binding to the site via a CaMK-dependent mechanism. Am J Physiol Endocrinol Metab 2008;294:E582-E588.

29 Khan Z, Akhtar M, Ekström TJ: HDAC inhibitor 4-phenylbutyrate preserves immature phenotype of human embryonic midbrain stem cells: implications for the involvement of DNA methyltransferase. Int J Mol Med 2011;28:977-983.

-30 Shin JH, Li RW, Gao Y, Baldwin R 6th, Li CJ: Genome-wide ChIP-seq mapping and analysis reveal butyrateinduced acetylation of H3K9 and H3K27 correlated with transcription activity in bovine cells. Funct Integr Genomics 2012;12:119-130.

- 31 Leturque A, Loizeau M, Vaulont S, Salminen M: Girard J. Improvement of insulin action in diabetic transgenic mice selectively overexpressing GLUT4 in skeletal muscle. Diabetes 1996;45:23-27. 